\title{
Perfil de dissolução in vitro de comprimidos de primaquina disponíveis para tratamento de malária no Brasil
}

\author{
In vitro dissolution profile of primaquine tablets available \\ for malaria treatment in Brazil
}

\author{
Letícia Norma Carpentieri Rodrigues $^{1}$, Sayuri Pereira Watanabe ${ }^{2}$ \\ e Humberto Gomes Ferraz ${ }^{2}$
}

\begin{abstract}
RESUMO
A ineficácia clínica de muitos medicamentos tem servido de alerta para estudos mais profundos sobre os componentes da formulação, processos empregados e características físico-químicas dos fármacos. 0 objetivo deste trabalho foi avaliar a liberação in vitro de comprimidos de fosfato de primaquina disponíveis no Brasil para tratamento da malária, e o desenvolvimento de novas formulações de liberação convencional. Embora os comprimidos de fosfato de primaquina estudados tenham sido aprovados pelos critérios propostos pela Farmacopéia Americana (2006) para o teste de dissolução, não apresentaram desempenho adequado para o perfil de dissolução, mostrando retenção do fármaco durante a liberação. Os resultados indicam a existência de problemas nos comprimidos de fosfato de primaquina analisados, podendo sugerir como um dos fatores responsáveis pelo aparecimento de resistência dos parasitas.
\end{abstract}

Palavras-chaves: Primaquina. Dissolução. Compatibilidade.

\section{ABSTRACT}

The clinical inefficacy of many medications has served to highlight the need for deeper studies on the formulation components, processes used and physicochemical characteristics of drugs. The objective of this study was to evaluate the in vitro release of primaquine phosphate from tablets available in Brazil for treating malaria, and the development of new formulations for conventional release. Although the primaquine phosphate tablets studied had been approved according to the criteria proposed by the United States Pharmacopoeia (2006) for the dissolution test, they did not present adequate dissolution performance characteristics, in that there was drug retention during the release process. The results indicate the existence of problems in the primaquine phosphate tablets analyzed, and it may suggest that this is one of the factors responsible for the appearance of parasite resistance.

Key-words: Primaquine. Dissolution. Compatibility.

Até o início da década de 60, era comum que se considerasse um medicamento eficaz clinicamente apenas assegurando-se o controle de qualidade, que incluía somente o conhecimento das propriedades físicas e físico-químicas do fármaco. A ineficácia clínica e, em alguns casos, intoxicações graves, serviram de alerta para estudos mais profundos sobre os componentes da formulação, processos empregados e características físicoquímicas dos fármacos ${ }^{17}$.

Dentre as formas farmacêuticas de uso oral, as formas farmacêuticas sólidas - particularmente os comprimidos - têm merecido destaque pela possibilidade de apresentarem problemas de biodisponibilidade.

Para obtenção do efeito terapêutico a partir de um medicamento na forma de comprimido, quando administrado por via oral, é necessário que o fármaco passe da forma de dosificação em que se encontra para outra em que seja diretamente absorvível.
Este processo, chamado de liberação, é anterior a absorção, e depende da dissolução do fármaco nos líquidos orgânicos. Assim, é fácil imaginar a importância dos ensaios de dissolução na avaliação do desempenho dos medicamentos na forma sólida.

0 estudo do processo de dissolução in vitro, empregando-se o teste de dissolução e perfil de dissolução tem sido utilizado como parâmetro crítico para determinar o desempenho e definir a qualidade da forma farmacêutica, servindo também como indicador preditivo da velocidade de absorção. A possibilidade de correlacionar os dados in vivo e in vitro é de valor inestimável para definir a qualidade biofarmacêutica de um medicamento, sendo útil no desenvolvimento de formulações, controle de qualidade e determinação de equivalentes farmacêuticos ${ }^{1}$.

A malária permanece, hoje, como um desafio para a humanidade, especialmente nos países em processo de desenvolvimento, atingindo cerca de $40 \%$ da população de

\footnotetext{
1. Departamento de Farmácia, Universidade Federal do Paraná, Curitiba, PR. 2. Faculdade de Ciências Farmacêuticas, Universidade de São Paulo, São Paulo, SP. Endereço para correspondência: Dr. Humberto Gomes Ferraz. Faculdade de Ciências Farmacêuticas/USP. Av. Lineu Prestes 580, 05508-900 São Paulo, SP. e-mail: sferraz@usp.br

Recebido para publicação em: 10/04/2007

Aceito em: 21/11/2007
} 
mais de 100 países, sendo considerada a mais insidiosa das parasitoses $^{12}$.

O Brasil, por meio da intensificação das ações de controle da malária, tem alcançado resultados positivos, entretanto fatores como o atraso e inadequação do diagnóstico e tratamento, resistência aos fármacos disponíveis, mantêm a cadeia epidemiológica do parasita. Segundo a Secretaria de Vigilância em Saúde (SVS/MS) em 2006 foram registrados 540.047 casos - a grande maioria na Amazônia legal ${ }^{15} 16$.

A primaquina, uma 8-aminoquinolina altamente ativa contra gametócitos de todas as espécies de malária humana e contra hipnozoítos do Plasmodium vivax, apresenta uso terapêutico como hipnozoiticida, usado nas infecções naturais por Plasmodium vivax, objetivando-se destruir as formas hipnozoíticas e como medicamento gametocitocida em infecções por Plasmodium falciparum (bloqueador de transmissão). No tratamento anti-hipnozoiticida, a primaquina é administrada conjuntamente com um esquizonticida sangüíneo ativo - como a cloroquina -, por um período de 7 a 14 dias na dose de $0,5 \mathrm{mg}$ de base $/ \mathrm{kg}$, enquanto a dose gametocitocida varia entre $0,50 \mathrm{a}$ $0,75 \mathrm{mg} / \mathrm{kg}$ em uma única dose $\mathrm{e}^{14}$.

A qualidade biofarmacêutica de formas farmacêuticas sólidas de administração oral tem sido intensamente estudada ${ }^{45}$. Vários trabalhos relativos à dissolução da cloroquina ${ }^{18}$ e quinino ${ }^{7}$ são citados na literatura, mas poucos trabalhos relativos ao antimalárico primaquina são encontrados. Kron, em 1996 ${ }^{11}$, investigou a qualidade dos comprimidos de fosfato de primaquina fornecidos à Namíbia para tratamento da malária, e constatou que os comprimidos continham menos que a metade da dose declarada no rótulo do produto. A pobre qualidade dos comprimidos foi relacionada com o aparecimento de resistência do parasito ao fármaco.

Assim, o objetivo deste estudo foi avaliar a liberação in vitro (dissolução) de formas farmacêuticas sólidas contendo fosfato de primaquina disponíveis para o tratamento da malária no Brasil e desenvolvimento de comprimidos de liberação convencional, obedecendo aos requisitos de qualidade biofarmacêutica.

\section{MATERIAL E MÉTODOS}

Substância química de referência e amostras. Fosfato de primaquina foi cedido pela Taixing Chemical Co, Ltd, Nanging, China (grau de pureza 98,7\%).

Um total de dez diferentes lotes de comprimidos, contendo $26,5 \mathrm{mg}$ de fosfato de primaquina (equivalente a $15 \mathrm{mg}$ de primaquina base) (produto $\mathrm{C}$ ), foi gentilmente cedido pela Secretaria de Vigilância em Saúde (SVS/MS).

Ensaios de dissolução. 0 método empregado para a realização do teste de dissolução seguiu critérios recomendados pela Farmacopéia Americana ${ }^{19}$ empregando aparelho de dissolução Hanson Research SR6. 0 meio de dissolução empregado foi $\mathrm{HCl}$ $0,01 \mathrm{M}$, para um volume de $900 \mathrm{~mL}$, sistema de agitação - aparato 2 (pá) e velocidade de agitação de 50rpm. A absorbância do meio foi avaliada ao final de 60 minutos em $224 \mathrm{~nm}$, sendo analisados seis comprimidos de cada lote de especialidade farmacêutica ou formulação desenvolvida. As concentrações foram obtidas com o auxílio de curva analítica previamente construída, garantindo linearidade no intervalo de 2 a $30 \mu \mathrm{g} \cdot \mathrm{mL}^{-1}(\mathrm{r}=0,9997)$. Os limites de quantificação e detecção foram $0,1179 \mu \mathrm{g} \cdot \mathrm{mL}^{-1}$ e $0,0354 \mu \mathrm{g}$. $\mathrm{mL}^{-1}$, respectivamente.

Um total de doze comprimidos de cada lote, contendo $26,3 \mathrm{mg}$ de fosfato de primaquina (equivalente a $15 \mathrm{mg}$ de primaquina base), foi submetido à dissolução sob condições idênticas àquelas descritas para o teste de dissolução ${ }^{19}$. Decorridos $1,3,5,10$, 20, 30, 40, 50, 60 e 70 minutos após o início do ensaio, foram retiradas alíquotas de $10 \mathrm{~mL}$ do meio de dissolução, fazendose, em seguida, a reposição deste com $\mathrm{HCl} 0,01 \mathrm{M}$ à mesma temperatura. As alíquotas retiradas foram centrifugadas por 15 minutos a 5.000rpm e posteriormente submetidas à leitura espectrofotométrica em 224nm (Beckman Coulter ${ }^{\circledR}$ UV-VIS mod. DU 640).

o perfil de dissolução das diferentes especialidades farmacêuticas analisadas foi traçado a partir da quantificação dos fármacos nas cubas de dissolução, em intervalos de tempo préestabelecidos. Os valores obtidos foram corrigidos, considerandose as alíquotas retiradas ${ }^{2}$ e expressos na forma de gráficos da porcentagem de fármaco dissolvido versus tempo.

Comparação dos perfis de dissolução. 0 parâmetro eficiência de dissolução (ED) foi calculado a partir das curvas de porcentagem dissolvida do fármaco versus tempo, através da razão entre a área sobre a curva (ASC) e a área total do gráfico (superfície), e expresso em porcentagem ${ }^{8}$. Os valores de ED obtidos foram submetidos à análise estatística usando-se análise da variância (ANOVA) ${ }^{3}$.

Calorimetria exploratória diferencial. Curvas DSC (differential scanning calorimetry) do fármaco puro e misturas binárias de fármaco-excipiente (1:1) foram obtidas por meio de célula DSC 2910 TA Instruments ${ }^{\circledR}$ sob atmosfera dinâmica de nitrogênio $\left(100 \mathrm{~mL} \cdot \mathrm{min}^{-1}\right)$; razão de aquecimento de $10^{\circ} \mathrm{C} \cdot \mathrm{min}^{-1}$, e registrados no intervalo de $40-400^{\circ} \mathrm{C}$. Foram empregadas cápsulas de alumínio seladas e massa de amostra de 3mg.

Seleção dos excipientes e planejamento das formulações. Estudos de compatibilidade fármaco-excipiente foram realizados usando calorimetria exploratória diferencial. Baseando-se nestes estudos os excipientes foram selecionados e procedeu-se o planejamento das formulações através de tratamento estatístico usando planejamento fatorial.

\section{RESULTADOS}

Avaliação das especialidades farmacêuticas. 0 teste de dissolução de comprimidos de fosfato de primaquina descrito na Farmacopéia Americana ${ }^{19}$ recomenda que não menos que $85 \%$ $(\mathrm{Q}+5 \%)$ do fármaco deverá ser dissolvido em 60 minutos. Todos os lotes avaliados cumpriram com as especificações no primeiro estágio $\left(\mathrm{S}_{1}\right)$ para o teste de dissolução, uma vez que a dissolução foi superior a $90 \%$ da quantidade declarada no rótulo após 60 minutos de ensaio. 
operfil de dissolução das amostras de comprimidos de fosfato de primaquina disponíveis para o tratamento de malária no Brasil apresentou problemas de liberação do fármaco (Figura 1).

A comparação dos perfis de dissolução utilizando-se o modelo independente de comparação, no qual os valores de eficiência de dissolução (ED) foram submetidos a tratamento estatístico, permitiu observar-se que em relação ao nível descritivo $\left(\mathrm{P}=6,5040 \times 10^{-6}\right)$ e valor de $\mathrm{F}$ calculado ao nível de significância de $5 \%(\mathrm{~F}=5,2284)$, os lotes de primaquina analisados não são iguais em média e homogêneos entre si.

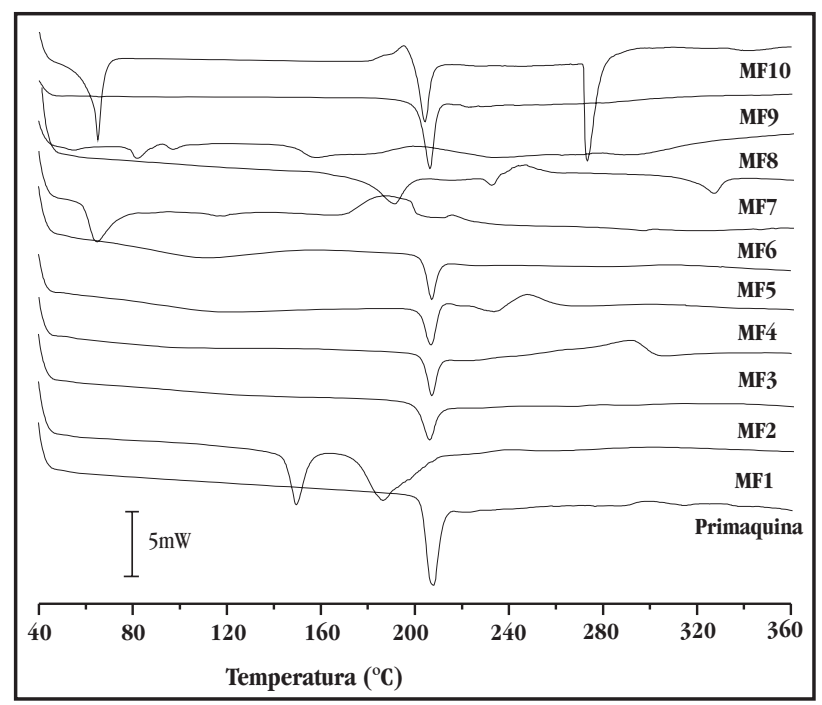

Figura 1 - Curvas DSC do fosfato de primaquina e da mistura-física 1:1 de fosfato de primaquina/lactose M200 (MF1), fosfato de primaquina/ dióxido de silício (MF2), fosfato de primaquina/celulose microcristalina (MF3), fosfato de primaquina/amido (MF4), fosfato de primaquina/ crospovidone USP/NF (MF5), fosfato de primaquina/estearato de magnésio (MF6), fosfato de primaquina/glicolato de amido sódico (MF7), fosfato de primaquina/lauril sulfato de sódio (MF8), fosfato de primaquina/talco (MF9) e fosfato de primaquina/ácido esteárico (MF10), obtidas sob atmosfera dinâmica de nitrogênio $\left(100 \mathrm{~mL}^{2} \mathrm{~min}^{1}\right)$ e razão de aquecimento de $10^{\circ} \mathrm{C} . \mathrm{min}^{1}$.

Estudos de compatibilidade fármaco/excipientes. Os estudos de compatibilidade do fármaco - fosfato de primaquina - e os excipientes - amido, celulose microcristalina, dióxido de silício, crospovidone, talco, lactose, glicolato de amido sódico, estearato de magnésio, lauril sulfato de sódio e ácido esteárico - realizados usando calorimentria exploratória diferencial, são apresentados na Figura 1.

Avaliação das formulações. As formulações desenvolvidas segundo projeto fatorial cumpriram com as especificações para o teste de dissolução no primeiro estágio $\left(\mathrm{S}_{1}\right)$, uma vez que a dissolução de ambos foi superior a $85 \%(Q+5 \%)$ da quantidade declarada no rótulo após 60 minutos de ensaio, conforme recomendado pela Farmacopéia Americana ${ }^{19}$. Avaliando-se as curvas de porcentagem dissolvida versus tempo - perfil de dissolução - (Figura 2), as formulações analisadas não apresentam diferenças significativas consideráveis e mostraram perfil de dissolução adequado à fármacos de pronta liberação.

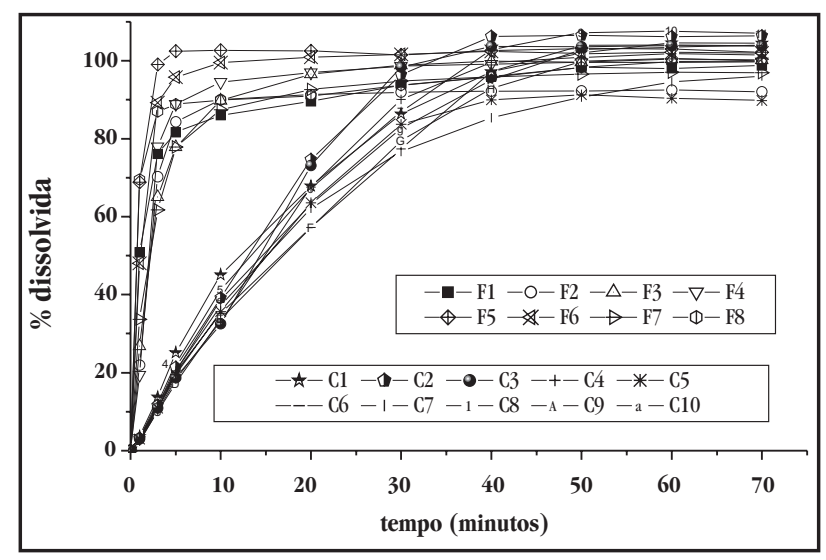

Valores obtidos para um total de 12 comprimidos para cada lote analisado.

Figura 2 - Perfis de dissolução de comprimidos de fosfato de primaquina para os diversos lotes das especialidades farmacêuticas (C) e formulações (F).

\section{DISCUSSÃO}

Avaliando-se as curvas de porcentagem dissolvida versus tempo - perfil de dissolução -, observou-se que, embora as amostras de fosfato de primaquina estudadas tenham sido aprovadas pelos critérios propostos pela Farmacopéia Americana ${ }^{19}$ para o teste de dissolução, mostraram retenção do fármaco, resultado não esperado para produtos de pronta liberação.

Durante estudos de formulação de novos produtos ou reformulação de produtos existentes, é vantajoso conhecer-se as possíveis interações entre fármacos e excipientes, que podem gerar mudanças na natureza química com perda acelerada da potência, formação de complexos, formação de eutéticos e conseqüiente alteração na estabilidade, solubilidade, absorção, e resposta terapêutica dos fármacos. DSC tem sido largamente usada para rápida avaliação de possíveis incompatibilidades entre os componentes da formulação através da comparação do comportamento térmico da substância pura com a mistura física 1:1, 1:3 ou 3:1 ou na razão de uso igual à formulaçã $0^{6}$.

Se a curva apresentada pela mistura física se superpõe àquela da substância química de referência, não há interação e, portanto, há compatibilidade físico-química entre fármaco e excipiente. Mudanças no aspecto do pico, temperatura onset $\left(T_{\text {onset }}\right)$ ou temperatura máxima de fusão $\left(T_{f}\right)$, altura relativas dos picos e, o aparecimento ou desaparecimento de um ou mais picos correspondentes aos componentes da mistura sugerem incompatibilidade ${ }^{6}$.

A comparação entre a sobreposição das curvas DSC referente à mistura física e fármaco e os dados relativos ao comportamento térmico da lactose (Figura 1), mostra que os eventos térmicos correspondentes à fusão e decomposição das substâncias individuais foram deslocados na mistura para temperatura mais baixas, indicando algum tipo de reação entre o fármaco e o excipiente que conduz às diferenças no perfil das curvas DSC da mistura. A incompatibilide da lactose com aminas primária e secundária é descrita por vários autores ${ }^{20}$.

Na mistura lauril sulfato de sódio/primaquina na proporção 1:1 (Figura 1) a curva DSC revelou eventos endotérmicos entre 50 e $160^{\circ} \mathrm{C}$, acima dessa temperatura observa-se eventos exotérmicos 
e endotérmicos seguidos, associados à decomposição térmica do material. 0 evento endotérmico de fusão do fosfato de primaquina não está sobreposto ao da mistura, sugerindo interação fármacoexcipiente.

0 glicolato de amido sódico exibe um evento endotérmico entre 50 e $180^{\circ} \mathrm{C}$, característico de desidratação. 0 mesmo é termicamente estável até $260^{\circ} \mathrm{C}$; porém acima dessa temperatura entre 270 a $340^{\circ} \mathrm{C}$ exibe um pico exotérmico referente à decomposição do material. Observando-se a curva DSC da mistura glicolato de amido sódico/primaquina na proporção 1:1 (Figura 1), foi possível observar um evento endotérmico em $191^{\circ} \mathrm{C}$ sugerindo interação fármaco-excipiente.

Miller e York ${ }^{13}$, em 1985, sugerem eventos endotérmicos entre 90 e $132^{\circ} \mathrm{C}$, referentes à fusão do estearato de magnésio. A curva DSC referente à mistura estearato de magnésio/primaquina na proporção 1:1 (Figura 1) revelou a etapa de desidratação nas temperaturas de $64 \mathrm{e} 117^{\circ} \mathrm{C}$, característica do estearato de magnésio. Porém, revelou eventos exotérmicos entre 165 e $230^{\circ} \mathrm{C}$, faixa de temperatura em que não ocorreram eventos térmicos para ambas as substâncias isoladamente. A comparação entre a sobreposição das curvas referentes à mistura física e fármaco, e os dados relativos ao comportamento térmico do estearato de magnésio, mostra que os eventos térmicos observados não são sobreponíveis em cada substância testada isoladamente, sugerindo interação entre o estearato de magnésio e 0 fosfato de primaquina. A incompatibilidade de estearato de magnésio com fármacos é citada por vários autores ${ }^{19}$.

Embora não se conheça a amplitude da interação do fármaco - fosfato de primaquina - com os excipientes - lactose anidra, glicolato de amido sódico, estearato de magnésio e lauril sulfato de sódio-, em nível clínico, deve ser lembrado que o medicamento citado, no mercado brasileiro, apresenta prazo de validade de quatro anos, o que poderia levar a consolidar as reações e para tanto, não sendo adequado 0 emprego desses excipientes na preparação de comprimidos.

As formulações contendo fosfato de primaquina preparadas baseando-se nos estudos de compatibilidade fármaco-excipiente cumpriram com as especificações para o teste de dissolução conforme recomendado pela Farmacopéia Americana ${ }^{19} \mathrm{e}$ apresentaram perfil de dissolução adequado, compatível com fármacos de pronta liberação.

As quinolinas antimaláricas absorvem luz na região do ultravioleta, UV-A (320 a 400nm) e UV-B (290 a 320nm), são fotoquimicamente instáveis. 0 estudo de degradação in vitro da primaquina demonstrou que a irradiação da primaquina em meio aquoso contendo oxigênio causa várias mudanças na cadeia lateral da molécula. 0s produtos de degradação fotoquímica da primaquina são potentes indutores de radicais livres ${ }^{90}$. Os produtos de degradação identificados para primaquina in vitro são diferentes dos metabólitos deste fármaco, mas há correlação da estabilidade in vitro e os efeitos adversos in vivo ${ }^{10}$.

Ensaios preliminares realizados pelo Ministério da Saúde, por intermédio da Rede Amazônica de Vigilância às Drogas Antimaláricas (RAVREDA), sobre a eficácia de fármacos constantes no Manual de Terapêutica da Malária, mostram resistência dos parasitos aos medicamentos e sugerem à qualidade dos medicamentos empregados como um dos fatores determinantes ${ }^{15}$.

Os resultados indicam a existência de problemas nas formulações de comprimidos de fosfato de primaquina disponíveis para tratamento da malária no Brasil, podendo sugerir como um dos fatores responsáveis pelo aparecimento de resistência dos parasitos. Tal fato reforça a necessidade da realização de estudos de pré-formulação e avaliação biofarmacêutica no desenvolvimento destes medicamentos.

\section{REFERÊNCIAS}

1. Arancibia A. Calidad biofarmacéutica estudios in vitro e in vivo. Acta Farmaceutica Bonaerense, La Plata 10: 123-133, 1991.

2. Aronson H. Correction factor for dissolution profile calculations. Journal of Pharmaceutical Sciences 82: 1190, 1993.

3. Bolton S, Bon C. Pharmaceutical statistics: practical and clinical applications. Drugs and the pharmaceutical sciences. Marcel Dekker, New York, vol: 135, p.215-259, 2004.

4. Ferraz HG, Consiglieri VO, Storpirtis S. Avaliação da cinética de dissolução de ampicilina em comprimidos comercializados no Brasil. Brazilian Journal of Pharmaceutical Sciences 34: 93-99, 1998.

5. Ferraz HG, Pinho JJRG, Uehara AC, Reis MT, Siguenaga AM. Estudo comparativo do perfil de dissolução de comprimidos de cloreto de potássio comercializados no Brasil. Brazilian Journal of Pharmaceutical Sciences 35: 95-99, 1999.

6. Ford JL, Timmins P. Pharmaceutical thermal analysis: techniques and applications. Ellis Harwood books in biological sciences. Series in pharmaceutical technology. Chichester, Ellis Horwood; Halsted Press, New York, 1989.

7. Kennedy RA, Stewart PJ. The effect of ph and ionic strength on the release of quinine adsorbed on to an insoluble sodium polyphosphate. Drug Development and Industrial Pharmacy 22: 713-719, 1996.

8. Khan KA, Rhodes CT. The concept of dissolution efficiency. The Journal of Pharmacy and Pharmacology 27: 48-49, 1975.

9. Kristensen S, Grinberg L, Tonnensen HH. Photoreactivity of biologically active compounds. XI. Primaquine and metabolites as radical inducers. European Journal of Pharmaceutical Science 5: 139-146, 1997.

10. Kristensen S, Grislingaas AL, Greenhill JV, Skjetne T, Tonnensen HH. Photochemical stability of biologically active compounds: V. Photochemical degradation of primaquine in an aqueous medium. International Journal of Pharmaceutics 100: 15-23, 1993

11. Kron MA. Substandard primaquine phosphate for US Peace Corps personnel. Lancet 348: 1453-1454, 1996.

12. Lalloo DG, Shingadia D, Pasvol G, Chiodini PL, Whitty CJ, Beeching NJ, Hill DR, Warrell DA, Bannister BA for the HPA Advisory Committee on Malaria Prevention in UK Travellers, UK malaria treatment guidelines. The Journal of Infection 54: 111-121, 2007.

13. Miller TA, York P. Physical and chemical characteristics of some highpurity magnesium stearate and palmitate powders. International Journal of Pharmaceutics 23: 55-67, 1985

14. Ministério da Saúde. Secretaria de Vigilância em Saúde. A Malária no Brasil. Brasilia: 2005. el em: http://portal.saude.gov.br/portal/arquivos/pdf/be_malaria_ 01_2005.pdf/ Acesso em: 21 set. 2007.

15. Ministério da Saúde. Secretaria de Vigilância em Saúde. Situação Epidemiológica da Malária no Brasil, ano de 2006. Brasilia, 2007. Disponível em: http://portal. saude.gov.br/portal/arquivos/pdf/folder_malaria_2007_web.pdf/ Acesso em: 20 set. $2007,2007$.

16. Ministério da Saúde. Fundação Nacional de Saúde. Manual de Terapêutica da Malária. Brasília: dezembro/2001. Disponível em: http://portal.saude.gov.br/ portal/arquivos/pdf/manu_terapeutica_malaria.pdf/ Acesso em: 20 set. 2007.

17. Storpirtis S. Biofarmácia e biofarmacotécnica: ensino e pesquisa. Infarma 2: $19-20,1993$. 
18. Tajerzadeh H, Rouini MR, Gharecolchian A. Relative bioavailability and pharmacokinetic parameters following administration of single oral dose (2x150 mg) of chloroquine tablet in healthy subjects. Drug Development and Industry Pharmacy 21: 2393-2402, 1995.
19. United States Pharmacopeia: USP 29/NF24. Supplement 1 Rockville: U.S. Pharmacopeial Convention, 2006.

20. Venkataram S, Khohlokwane M, Wallis SH. Differential scanning calorimetry as a quick scanning technique for solid state stability studies. Drug Development and Industrial Pharmacy 21:847-855, 1995. 